\title{
INSIGHTS
}

\section{The Doctor's Dilemma: lessons from GB Shaw in a modern pandemic COVID-19}

Eleanor J. Molloy (iD) $1,2,3,4$

In the current COVID 19 pandemic, the only treatments are supportive as no definitive pharmacological intervention is available. The heterogeneity of the immune response in different patient groups is clear with less severe illness in children. Understanding these disparities is particularly important as severely affected patients with COVID19 cannot always be predicted before they experience a cytokine storm and multiorgan dysfunction. Over 100 years ago, the concept of individualised immunotherapy was introduced by Sir Almroth Wright and immortalised in GB Shaw's play The Doctor's Dilemma. Shaw's play The Doctor's Dilemma explores the issues of private medical practice, equality of health care delivery, rationing of scarce resources (intensive care) and high-risk therapies. The play also describes the dilemma of rationing of resources and selecting the correct patient for new experimental therapies. Immunological theories of the time are now reflected in current understanding of inflammatory responses in sepsis and immunomodulation during the COVID19 pandemic.

Pediatric Research (2021) 89:701-703; https://doi.org/10.1038/s41390-020-0927-1

\section{INTRODUCTION}

The rapidly progressing coronavirus disease 2019 (COVID-19) pandemic has raised issue such as rationalising the use of intensive care beds and the emergence of immunotherapies. Both are described in the play The Doctor's Dilemma over 100 years ago. ${ }^{1}$ Immunotherapy is described for tuberculosis (TB) in the preantibiotic era with the suggestion of using antitoxins. Casadevall et al. have proposed the use of convalescent serum in COVID-19 as technique pioneered in the early 1900 s. $^{2}$ Doctors across the world have described the rationing of resources such as critical care beds and ventilators, which is the key discussion point in The Doctor's Dilemma as they struggle with "who to save". Rediscovering the work of early immunologists may give important insights into the management of COVID-19.

\section{Context of The Doctor's Dilemma}

The Doctor's Dilemma was written by George Bernard Shaw in 1902. ${ }^{1}$ The antihero Sir Colenso Ridgeon is a prominent physician-scientist of the time and is considered a gentle caricature of Shaw's friend Sir Almroth Wright, a prominent bacteriologist and immunologist (1861-1947). Almroth Wright attended Medical School at Trinity College Dublin and, in 1896, developed first successful vaccine against typhoid. This vaccine was widely used in World War I credited with saving $>100,000$ lives. In 1902, he started the inoculation department at St Mary's Hospital, today known as the Wright-Fleming Institute. This laboratory also included Leonard Colebrook who was vital in the chemotherapeutic conquest of puerpual sepsis ${ }^{3}$ and Sir Alexander Fleming who discovered penicillin there. ${ }^{4}$ Sir Almroth Wright warned of antibiotic resistance and advocated preventative medicine and logic in medical education. However, he was a controversial figure especially with the publication of "The Unexpurgated Case against Woman Suffrage" in 1913. The play makes many references to immunological theories of the day including opsonisation, phagocytosis and individualised immunotherapy.

\section{"Stimulate the phagocytes, drugs are a delusion"}

This quote from the play comments on the treatment of TB in the pre-antibiotic era when immunotherapy was pioneered. "Nature has provided, the white corpuscles as you call the -the phagocytes a we call them- a natural means of devouring and destroying all disease germs. There is at bottom only one genuinely scientific treatment for all diseases, and that is to stimulate the phagocytes, drugs are a delusion". Antibiotic resistance was predicted by both Fleming and Wright on the discovery of penicillin. Penicillin resistance to Staphylococcus aureus was first observed in 1947. There was a high mortality prior to the introduction of penicillin in the 1940s. There are no medications for COVID-19 although many are in clinical trials including azithromycin and corticosteroids. ${ }^{7}$ At present, healthcare workers are relying on supportive intensive care. However, recent publications suggest the use of immunotherapy including human antiserum and recombinant anti-inflammatory medications like tocilizumab. The use of human convalescent serum is proposed by Casadevall et al. ${ }^{2}$ This passive antibody treatment may result in viral inactivation, possibly enhanced phagocytosis cellular cytotoxicity or immunomodulation. Antibodies could be produced from those who recover from COVD-19 and ultimately humanised monoclonal antibodies derived. Casadevall and Pirofski suggest the use of passive antibody therapy either as prophylaxis for key healthcare workers or acutely as rescue

\footnotetext{
${ }^{1}$ Paediatrics, Trinity College, the University of Dublin, Dublin, Ireland; ${ }^{2}$ Trinity Translational Medicine Institute (TTMI) \& Trinity Research in Childhood Centre (TRICC), Trinity College Dublin, Dublin, Ireland; ${ }^{3}$ Paediatrics \& Neonatology, Children's Hospital Ireland (CHI) at Tallaght \& Crumlin, Dublin, Ireland and ${ }^{4}$ Coombe Women and Infants University Hospital, Dublin, Ireland

Correspondence: Eleanor J. Molloy (eleanor.molloy@tcd.ie)
}

Received: 31 March 2020 Accepted: 1 April 2020

Published online: 28 April 2020 
702

treatment for patients in intensive care. ${ }^{2}$ Recently, in a case series of five critically ill patients with COVID-19 and acute respiratory distress syndrome, convalescent plasma with neutralising antibody resulted in improvement in clinical outcomes. ${ }^{8}$ Innate immune function including opsonisation and phagocytosis are crucial to effectively resolve infection. An opsonin is any molecule that acts as a binding enhancer for the process of phagocytosis for example by coating the negatively charged molecules on the membrane and the name is derived from the Greek "I prepare victuals for". Both the membranes of a phagocytising cell and its target have a negative charge, making it difficult for the two cells to come close together. Most phagocytic binding cannot occur without opsonisation of the antigen. Examples of opsonin molecules include antibodies: immunoglobulin $G(\lg G)$ and $\lg A$, and components of the complement system: $\mathrm{C} 3 \mathrm{~b}, \mathrm{C} 4 \mathrm{~b}$, and $\mathrm{iC} 3 \mathrm{~b}$.

Sir Ridgeon, the antihero in the play describes opsonisation as follows: "Opsonin is what you butter the disease germs with to make your white corpuscles eat them... What it comes to in practice is this. The phagocytes won't eat the microbes unless the microbes are nicely buttered for them".

Theoretically, infectious morbidity and mortality could be reduced by the administration of intravenous $\mathrm{lg}$. This therapy has been suggested in COVID19 although there is an absence of clinical trials to support this at present. ${ }^{9,10}$

Systemic inflammatory and compensatory anti-inflammatory response syndromes

Ridgeon's theory of opsonisation (buttering) and pro- and antiinflammatory responses during infection mirror our current concepts of immune responses in sepsis. In Shaw's play, Sir Ridgeon advocates the activation of phagocytosis and describes his theories as follows: RIDGEON: but my discovery is that the manufacture of that butter, which I call opsonin, goes on in the system by ups and downs, Nature being always rhythmical you know, and what the inoculation does is to stimulate the ups or downs as the case may be.... Inoculate when the patient is in the negative phase and you kill; inoculate when the patient is in the positive phase and you cure".

This may be one of the first description of the proinflammatory systemic inflammatory response syndrome (SIRS) and its counterpart the compensatory anti-inflammatory response (CARS). The domination of either the CARS or SIRS response is associated with increased morbidity and mortality. Adjunctive immunomodulatory treatments for sepsis seek to balance these responses and restore homoeostasis. ${ }^{11,12}$ However, discovering which inflammatory phase is dominant in the patient at a certain time point remains difficult and hinders appropriate therapeutic immunomodulation. ${ }^{7}$

Anti-inflammatory treatment can increase mortality due the constantly dynamic nature of the sepsis continuum including both SIRS and CARS. The single "magic bullet" for sepsis is elusive for this reason and stratifying patients according to their immune response seems more effective. Following caecal ligation and puncture (CLP) model of sepsis in a murine model, mortality was increased in mice pretreated with interleukin receptor antagonist (IL-1ra) increases mortality after CLP. ${ }^{13}$

Individualised immunotherapy

The opsonin index: "Send a drop of the patient's blood to the laboratory at St. Anne's; and in fifteen minutes I'll give you his opsonin index in figures. If the figure is one, inoculate and cure: if it's under point eight, inoculate and kill". "To inject a vaccine into a patient without first testing his opsonin is as near murder as a respectable practitioner can get".

HLA-DR expression on monocytes might constitute a useful indicator of the immunological status of the individual patient with sepsis and a guide for treatment. Patients with CARS, as manifested by low HLA-DR expression, might benefit from immunostimulants, while patients with SIRS and normal or high monocyte HLA-DR expression should receive treatment directed to interfere with pro-inflammatory pathways. ${ }^{14}$ Osuchowski et al. $^{15}$ stratified mice using IL-6 as an early biomarker and showed that targeted immunosuppression decreased mortality in mice with predicted poor outcome. However, Panacek at al. ${ }^{16}$ have shown that using IL-6 to stratify patients receiving antitumour necrosis factor therapy $(n=2634)$ showed an decrease in mortality. A currently registered Chinese study of tocilizumab in COVID-19 management stratifies patients by IL-6 levels (ChiCTR2000029765). ${ }^{17}$

\section{CONCLUSION}

In Shaw's time (pre-antibiotics), the only solution was to stimulate the phagocytes. However, immunomodulation is still not an exact science in sepsis although carefully stratified individualised care is well established in cancer. The advent of antibiotic resistance has led to a resurgence in interest in immunomodulatory adjunctive therapy. Interestingly, this mirrors the research of Sir Almroth Wright and his research group in St. Marys over 100 years ago.

"I'm not belittling your discovery. Most discoveries are made regularly every fifteen years; and it's fully a hundred and fifty since yours was last made. That's something to be proud of". Therefore, Sir Almroth Wright in his time known as Sir Always Wrong was probably Sir Almost Wright.

\section{ACKNOWLEDGEMENTS}

This work was supported by Health Research Board, Ireland.

\section{ADDITIONAL INFORMATION}

Competing interests: The author declares no competing interests.

Publisher's note Springer Nature remains neutral with regard to jurisdictional claims in published maps and institutional affiliations.

\section{REFERENCES}

1. Shaw, G. B. The Doctor's Dilemma. Preface. 1913 (Penguin Books, Baltimore, 1941).

2. Casadevall, A. \& Pirofski, L. A. The convalescent sera option for containing COVID19. J. Clin. Invest. 130, 1545-1548 (2020).

3. Dunn, P. M. Dr Leonard Colebrook, FRS (1883-1967) and the chemotherapeutic conquest of puerperal infection. Arch. Dis. Child. Fetal Neonatal Ed. 93, F246-F248 (2008).

4. Brown, K. Penicillin Man: Alexander Fleming and the Antibiotic Revolution (Sutton Publishing limited, 2004).

5. Colebrook, L. Almroth Wright. Provocative Doctor and Thinker (Heinemann Medical, London, 1954).

6. Dunnill, M. The Plato of Praed Street: The Life and Times of Almroth Wright (Royal Society of Medicine Press, London, 2000).

7. Molloy, E. J. et al. Neonatal sepsis: need for consensus definition, collaboration and core outcomes. Pediatr. Res. https://doi.org/10.1038/s41390-020-0850-5 (2020).

8. Shen, C. et al. Treatment of 5 critically ill patients with COVID-19 with convalescent plasma. JAMA https://doi.org/10.1001/jama.2020.4783 (2020).

9. Li, G. et al. Coronavirus infections and immune responses. J. Med. Virol. 92, 424-432 (2020)

10. Fu, Y., Cheng, Y. \& Wu, Y. Understanding SARS-CoV-2-mediated inflammatory responses: from mechanisms to potential therapeutic tools. Virol. Sin. https://doi. org/10.1007/s12250-020-00207-4 (2020).

11. Bone, R. C. Sir Isaac Newton, sepsis, SIRS, and CARS. Crit. Care Med. 24, 1125-1128 (1996).

12. Hotchkiss, R. S. \& Karl, I. E. The pathophysiology and treatment of sepsis. N. Engl. J. Med. 348, 138-150 (2003).

13. Ashare, A. et al. Anti-inflammatory response is associated with mortality and severity of infection in sepsis. Am. J. Physiol. Lung Cell. Mol. Physiol. 288, L633-L640 (2005). 
The Doctor's Dilemma: lessons from GB Shaw in a modern pandemic COVID-19 EJ Molloy

14. Haveman, J. W. et al. The central role of monocytes in the pathogenesis of sepsis: consequences for immunomonitoring and treatment. Neth. J. Med. 55, 132-41. (1999).

15. Osuchowski, M. F., Connett, J., Welch, K., Granger, J. \& Remick, D. G. Stratification is the key: inflammatory biomarkers accurately direct immunomodulatory therapy in experimental sepsis. Crit. Care Med. 37, 1567-1573 (2009).
16. Panacek, E. A. et al. Efficacy and safety of the monoclonal anti-tumor necrosis factor antibody $F\left(a b^{\prime}\right) 2$ fragment afelimomab in patients with severe sepsis and elevated interleukin-6 levels. Crit. Care Med. 32, 2173-2182 (2004).

17. Chinese Clinical Trial Registry. A multicenter, randomized controlled trial for the efficacy and safety of tocilizumab in the treatment of new coronavirus pneumonia (COVID-19). http://www.chictr.org.cn/showprojen.aspx (2020). 